\section{Gut abgeschnitten}

C riseure leiden wie kaum eine andere Seit Jahren versucht die Berufsgenossenschaft durch Präventionsmaßnahmen wie den Erlass entsprechender Arbeitsvorschriften oder das Verbot von Glyzerylmonothioglykolat 1995 die Situation zu verbessern. Offenbar mit Erfolg, wie eine Untersuchung der Universität Heidelberg belegt. Die Arbeitsmediziner hatten von 1990 bis 1999 jährlich die Inzidenz berufsbedingter Dermatosen bei Friseuren in Franken und der Oberpfalz dokumentiert. Die Anzahl der Neuerkrankungen war über die Jahre kontinuierlich gesunken von 194 pro 10.000 Friseure im Jahr 1990 auf nur noch 18 im Jahr 1999.

Dickel H et al. BMJ 2002; 324: 1422-3

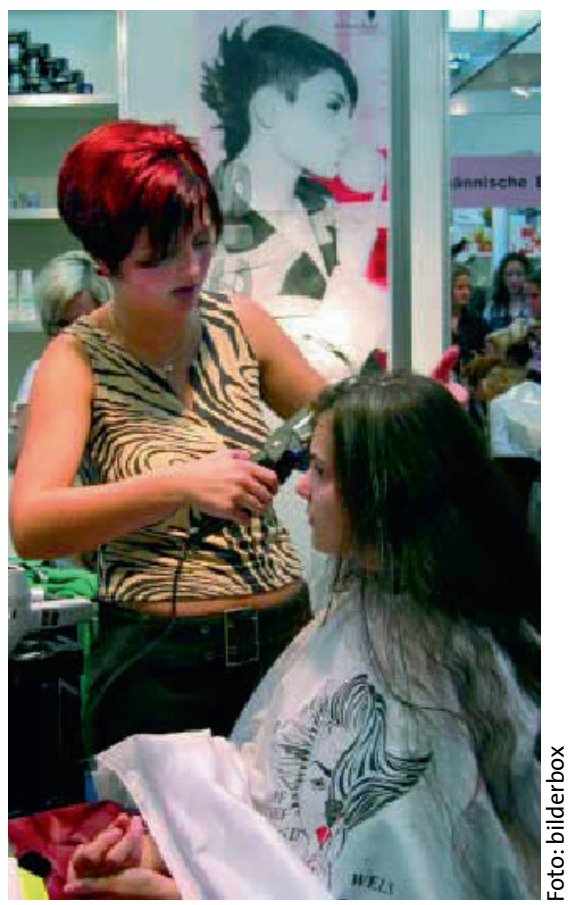

Digitale Peak-Flow-Kontrolle

A lle bisherigen Studien, die den $\mathrm{Zu}$ sammenhang zwischen häuslichen Peak-Flow-Selbstmessungen und der Qualität der Asthmakontrolle überprüfen sollten, hatten mit der geringen $\mathrm{Pa}$ tienten-Compliance von deutlich unter $50 \%$ zu kämpfen. Australische Forscher schickten jetzt ein digitales Peak-FlowMeter mit Speicherfunktion ins Rennen und konnten nach 72 Wochen Studiendauer eine Compliance von $89 \%$ dokumentieren. $\mathrm{Ob}$ dieser außergewöhnlich hohe Wert dem Spaß an dem High-Tech-Gerät zu verdanken ist, oder vielmehr der Speicherfunktion, die ein nachträgliches Erfinden von Messwerten unmöglich macht, konnten die Pneumologen auch nicht schlüssig beantworten.

Reddel HK et al. BMJ 2002; 324: 146-7

\title{
Interaktive Immunzellen
} der US Postal Service dazu übergegangen, Briefe und Pakete mit Elektronenstrahlen zu sterilisieren, um eventuelle Erreger abzutöten. Eine Behandlung, die auch per Post verschickten Allergenlösungen nicht gut bekommt, wie Allergologen der US-Army in einer

Testreihe jetzt herausgefunden haben. Die molekulare Zusammensetzung der bestrahlten Substanzen blieb nicht erhalten, die antigenen Epitope waren verändert oder vollständig verschwunden.

isl

Katial RK et al. J Allergy Clin Immunol

2002; 110: 215-9

\section{Katzenhaare im Klassenzimmer}

D as GSF-Forschungszentrum in Neuherberg erfasste die Daten von insgesamt 1.893 Schülern in Sachsen-Anhalt: $36,1 \%$ der Kinder hatten regelmäßig Kon-

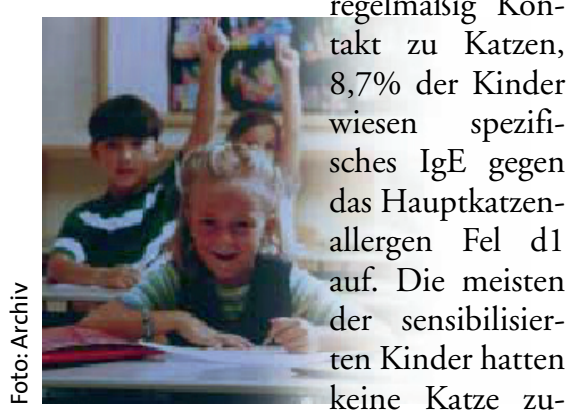

hause und waren nach Aussage der Eltern auch noch nie länger in Kontakt mit Katzen gekommen. Allerdings stieg bei diesen Kindern ohne Katze im Haushalt die Häufigkeit einer Sensibilisierung gegen Katzenhaare mit der Anzahl der Klassenkameraden, die Katzen zu Hause hatten. Die Epidemiologen ziehen den Schluss, dass die Menge der durch die Klassenkameraden eingeschleppten Katzenallergene offenbar für eine Sensibilisierung ausreichend groß ist.

isl

Ritz BR et al. Allergy 2002; 57: 357-61
- inem Forscherteam von der Harvard mit Hilfe grün markierter Moleküle des Major Histocompatibility Complex (MHC) zum ersten $\mathrm{Mal}$ live die Interaktion von dendritischen Zellen mit TZellen unter dem Mikroskop zu beob-

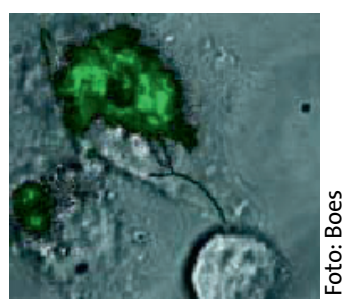
achten. Die

von der dendritischen Zelle synthetisierten MHC gruppieren sich im Zytoplasma in großer Zahl um phagozytierte Antigene. Es entsteht ein AntigenMHC-Komplex, den die dendritische Zelle bei Kontakt mit einem T-Lymphozyten durch in Minutenschnelle aktiv ausgebildete, bis zu $50 \mu \mathrm{m}$ lange Mikrotubuli (Bild) übergibt.

Boes $M$ et al. Nature 2002; 418: 983-8 